Distribution Category:

Magnetic Fusion Reactor Materials

(UC-423)

ANL/FPP/TM-271

ARGONNE NATIONAL LABORATORY

9700 South Cass Avenue

Argonne, Illinois 60439-4801

\title{
MODELING PLASMAMMATERIAL INTERACTIONS DURING A TOKAMAK DISRUPTION
}

\author{
by \\ Ahmed Hassanein \\ Fusion Power Program/Energy Technology Division \\ Isak Konkashbaev \\ Troitsk Institute for Innovation, Russia
}

October 1994

Work supported by the Office of Fusion Energy U.S. Department of Energy under Contract W-31-109-Eng-38 


\section{DISCLAIMER}

This report was prepared as an account of work sponsored by an agency of the United States Government. Neither the United States Government nor any agency thereof, nor any of their employees, make any warranty, express or implied, or assumes any legal liability or responsibility for the accuracy, completeness, or usefulness of any information, apparatus, product, or process disclosed, or represents that its use would not infringe privately owned rights. Reference herein to any specific commercial product, process, or service by trade name, trademark, manufacturer, or otherwise does not necessarily constitute or imply its endorsement, recommendation, or favoring by the United States Government or any agency thereof. The views and opinions of authors expressed herein do not necessarily state or reflect those of the United States Government or any agency thereof. 


\section{DISCLAIMER}

Portions of this document may be illegible in electronic image products. Images are produced from the best available original document. 


\section{TABLE OF CONTENTS}

Page

ABSTRACT

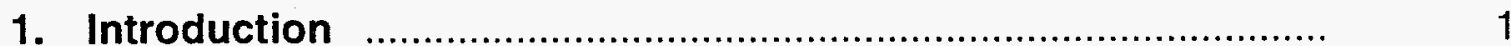

2. Physical Model ......................................................................

2.1 Material Thermal Evolution ……….........................................

2.2 Vapor Plasma Hydrodynamics ….........................................

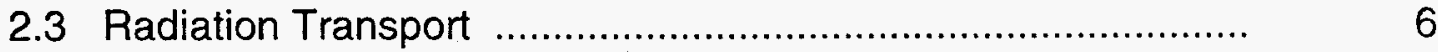

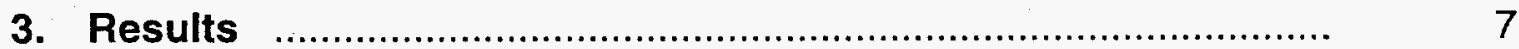

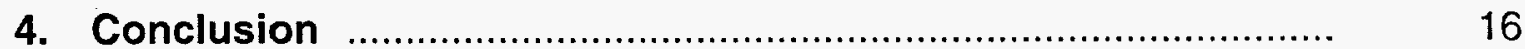

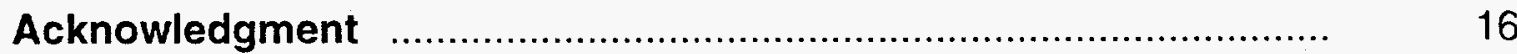

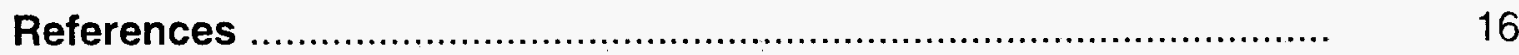




\section{LIST OF FIGURES}

Figure No.

Page

1 Schematic illustration of various interaction zones and processes during a plasma disruption.

2 Change in beryllium surface temperature, melt layer, and eroded thickness with time, following exposure to plasma electrons.

3 Relationship of target and vapor temperature to distance at different times during disruption.

$4 \quad$ Effect of plasma electron kinetic energy on beryllium erosion rate.

5 Change in beryllium vapor front temperature with time. ...

Effect of higher disruption energy densities on carbon erosion rate.

$7 \quad$ Effect of higher disruption energy densities on beryllium erosion rate.

Effect of higher disruption energy densities on carbon vapor-front temperature.

Effect of higher disruption energy densities on beryllium vapor-front temperature.

10 Erosion rate of beryllium and carbon at various disruption energy densities. 


\title{
MODELING PLASMA/MATERIAL INTERACTIONS \\ DURING A TOKAMAK DISRUPTION
}

\section{A. Hassanein and I. Konkashbaev}

\begin{abstract}
Disruptions in tokamak reactors are still of serious concern and present a potential obstacle for successful operation and reliable design. Erosion of plasmafacing materials due to thermal energy dump during a disruption can severely limit the lifetime of these components, therefore diminishing the economic feasibility of the reactor.
\end{abstract}

A comprehensive disruption erosion model which takes into account the interplay of major physical processes during plasma-material interaction has been developed. The initial burst of energy delivered to facing-material surfaces from direct impact of plasma particles causes sudden ablation of these materials. As a result, a vapor cloud is formed in front of the incident plasma particles. Shortly thereafter, the plasma particles are stopped in the vapor cloud, heating and ionizing it. The energy transmitted to the material surfaces is then dominated by photon radiation. It is the dynamics and the evolution of this vapor cloud that finally determines the net erosion rate and, consequently, the component lifetime. The model integrates with sufficient detail and in a self-consistent way, material thermal evolution response, plasma-vapor interaction physics, vapor hydrodynamics, and radiation transport in order to realistically simulate the effects of a plasma disruption on plasma-facing components. Candidate materials such as beryllium and carbon have been analyzed. The dependence of the net erosion rate on disruption physics and various parameters was analyzed and is discussed.

\section{Introduction}

The behavior of plasma-facing materials (PFMs) during a major disruption is critical for successful and reliable operation of a reactor. During the rapid thermal quench $(\approx 100 \mu \mathrm{s})$ of a disruption, energetic plasma electrons and ions strike parts of the divertor plate, depositing energy densities of $10-100 \mathrm{MJ} / \mathrm{m}^{2}$ and thus causing surface melting and ablation. If all of the incident energy were to be 
deposited entirely in the PFM, extensive ablation would severely limit the lifetime of the divertor plate and critically diminish the economic feasibility of the reactor.

However, it is expected that the initial wave of the ablated material will form a vapor cloud in front of the incoming plasma particles, thereby reducing the energy flux to facing-material surfaces. This vapor shielding effect would then significantly reduce the eroded thickness of these materials and consequently prolong its lifetime substantially. Several analyses of this vapor shielding effect have been performed [1-7]. Some previous work focused on one or two separate areas of the various processes involved during plasma-material interaction. Other work has focused separately on issues such as radiation transport [8], simulation experiments [9], and magnetic field effects [10].

Three major areas must be investigated to correctly evaluate material response during a plasma disruption. These are: target material thermodynamic response, vapor cloud hydrodynamics, and radiation transport. Figure 1 is a schematic illustration of the various interaction zones and processes that occur during the plasma/material interaction following a disruption. Basically this problem involves three moving boundaries: the vapor front, the receding surface, and the solid-liquid interface. These three moving boundaries are interdependent and a complete solution should link them dynamically and simultaneously. A comprehensive model is developed in which the interplay of the major physical processes during the plasma/material interaction are taken into account. Models for thermal evolution of a material, plasma/vapor interaction physics, vapor hydrodynamics, and radiation transport have been developed, integrated, and perfected in a self-consistent way in sufficient detail to realistically simulate the effect of a disruption on plasma-facing components. Candidate PFMs such as beryllium and carbon were considered in this analysis. The dependence of the net erosion rate on the characteristics of the plasma-vapor interaction zone for both plasma ions and electrons was analyzed and is discussed. The effect of uncertainties in reactor disruption conditions on the net erosion rate was also analyzed. 


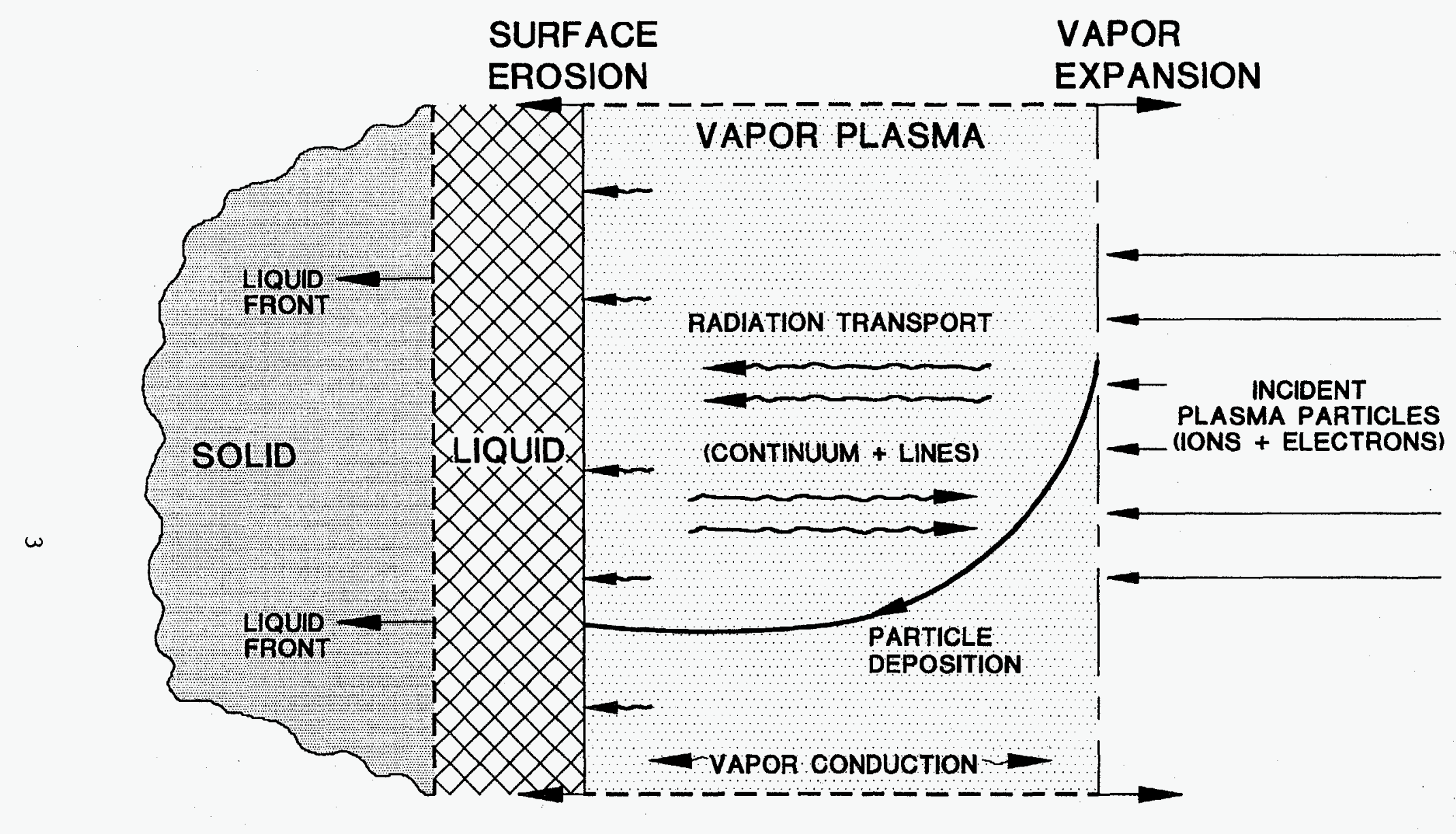

Fig. 1 Schematic illustration of various interaction zones and processes during a plasma disruption. 


\section{Physical Model}

\subsection{Material Thermal Evolution}

The thermal response of the PFM is calculated by solving a time-dependent heat conduction equation in one-or two-dimensional coordinates [11]. For simplicity, the heat conduction equation in the one-dimensional form ( $Z$ being the direction perpendicular to the surface) can be given by

$$
\rho c_{p} \frac{\partial T}{\partial t}=\nabla \cdot(K \nabla T)+\dot{q}(Z, t)
$$

where $\rho$ is the density, $c_{p}$ is the specific heat, $K$ is the thermal conductivity, and $\dot{q}$ is the volumetric energy deposition rate of the energetic plasma ions and electrons. All thermophysical properties are assumed to be a function of local temperature. Surface temperature was determined by both the boundary conditions and the evaporation process. The correct boundary condition at the surface requires that the incident energy be partitioned into conduction, melting, and evaporation. The boundary condition at the surface can then be given by

$$
-K \frac{\partial T}{\partial Z}(0, t)=q_{g a s}+q_{r a d}-q_{\text {evap }}
$$

where $\mathrm{qgas}_{\mathrm{g}}$ is the net heat flux from the near-wall vapor zone; $\mathrm{q}_{\mathrm{rad}}$ is the radiation heat flux absorbed at the material surface; and qevap is the evaporated heat flux, as determined by the enthalpy of evaporation. The evaporation flux leaving the surface is calculated in detail from models developed under nonequilibrium conditions $[2,18]$. Several boundary conditions can be used at the back surface of the material. The model we developed can also be used to analyze the response of various layers of materials such as coating, substrate, and intermediate layers, with each layer having its own boundary conditions. This is important in studying the effect of disruption on substrate structural materials under certain disruption conditions [12].

The volumetric energy deposition function $\dot{q}(Z, t)$ is calculated, in the condensed target material, with detailed models that include the slowing-down physics of plasma particles (both ions and electrons) in various target materials 
[9]. Phase change of metallic materials, when temperature exceeds their melting points are calculated using previously developed models [13].

The net heat flux $q_{g a s}$, due to particle transport from the near-surface vapor to the target, is described by a free-streaming energy transport term according to $[14,15]$

$$
q_{\text {gas }}=\alpha \frac{1}{4} n_{i} v_{i} \frac{3}{2} k\left(T_{i}-T_{s}\right)
$$

where $v_{i}$ is ion thermal velocity, $T_{i}$ is ion temperature, $T_{S}$ is the target surface temperature, and $\alpha$ is a collisional modification factor (accommodation coefficient $\alpha \approx 0.2$ ) of the free-streaming energy transport. The free-streaming term is more important for higher Z-materials such as tungsten, where the vapor near the target is rather dense [6]. The electron free-streaming term is neglected because a negative potential is assumed at the target surface.

\subsection{Vapor Plasma Hydrodynamics}

As vapor leaves the surface of a solid or liquid material, accumulates, and expands into the vacuum vessel, it interacts with incoming plasma particles. The plasma particles then deposit part of their energy into the vapor and the remainder into the condensed phase of the surface material behind the vapor. More vapor is then produced and thus more plasma energy is deposited into the vapor. Shortly thereafter, the plasma particles completely stop in the vapor, and produce intense bulk vapor heating and vapor ionization. Further heating of the PFM is only from vapor thermal radiation, vapor thermal conduction, freestreaming energy, and enhanced plasma radiation losses.

The extent of vapor expansion (one of the moving boundaries) into the vacuum vessel is determined by solving the vapor hydrodynamic equations for conservation of mass, momentum, and energy:

$$
\begin{aligned}
& \frac{\partial \rho}{\partial t}+\nabla \cdot(\rho V)=0, \\
& \rho \frac{\partial V}{\partial t}+\nabla P=0,
\end{aligned}
$$


and

$$
\frac{\partial \mathrm{E}}{\partial \mathrm{t}}+\nabla \cdot(\mathrm{EV})+\mathrm{P} \nabla \cdot \mathrm{V}=\nabla \cdot(\mathrm{K} \nabla \mathrm{T})+\nabla \cdot \mathrm{Q}_{\mathrm{r}}+\nabla \cdot \mathrm{Q}_{\mathrm{b}}
$$

where $V$ is the vapor velocity, $K$ is the vapor conductivity, $Q_{r}$ is the radiation flux, and $Q_{b}$ is the plasma particle beam flux. All variables of these equations are both time and space dependent. The solution of these equations is approximated by finite difference methods described in [15]. The plasma beam particle flux deposited in the vapor is calculated by similar methods for slowing down in a cold target, i.e., by inelastic and elastic collisions; however an additional stopping mechanism is used which arises from the free electrons due to ionization. This free electron stopping term can substantially shorten the range of both plasma ions and electrons in the ionized vapor leading to high energy deposition rate at the front of the vapor zone.

\subsection{Radiation Transport}

It is the radiation transport that will finally determine the net energy flux to PFMs and, consequently, determine the net erosion rate and lifetime of the divertor plate. It is then important to correctly model the radiation transport for a wide range of vapor conditions. For quasistationary conditions, the transport equation for the radiation has the form

$$
\vec{\Omega} \nabla \mathrm{I}_{v}=\varepsilon_{v}-\kappa_{v} \mathrm{I}_{v},
$$

where $I_{v}$ is radiation intensity, $v$ is frequency, $\varepsilon_{v}$ is vapor emissivity, $\vec{\Omega}$ is the solid angle, and $K_{v}$ is the absorption coefficient. Several methods are available for solving radiation transport equations. However, the most convenient one is the so-called forward-reverse method [16]. The main advantage of this method is a better description of both the optically thin and the optically thick plasma conditions [17]. Other popular methods, such as diffusion approximation are valid only for optically thick plasma conditions. The forward-reverse method treats the photon flux moving to the right (forward) $\mathrm{I}_{v}^{+}$separately from the photon flux moving to the left (reverse) $\mathrm{I}_{v}^{-}$. Assuming one-dimensional plane geometry, and after integrating over the angles, the radiation fluxes in the forward and reverse direction are calculated for each vapor zone as 


$$
\frac{1}{2} \frac{\mathrm{d} \mathrm{I}_{v}^{ \pm}}{\mathrm{dx}}=\varepsilon_{v}-\kappa_{v} \mathrm{I}_{v}^{ \pm}
$$

In these calculations, the radiation fluxes are composed of two separate components, continuum radiation flux $\mathrm{I}_{\mathrm{c}_{v}}$ and lines radiation flux $\mathrm{I}_{\ell_{v}}$, so that

$$
\mathrm{I}_{v}^{ \pm}=\mathrm{I}_{\mathrm{cv}}^{ \pm}+\mathrm{I}_{\ell v}^{ \pm}
$$

Therefore, the most intense lines are treated separately using the Collisional Radiative Equilibrium (CRE) method. This is because the vapor cloud plasma is not in local thermodynamic equilibrium (LTE). The atomic level populations are not obtained from the Saha equation and Boltzmann distributions. A set of rate equations is therefore solved for the populations of each individual atomic level. The less intense lines are combined with the continuum radiation. The most intense lines are usually $<100$ lines for each of the beryllium and carbon materials. Each line is approximated by about 10-20 photon energy groups, depending on line shape and width. Doppler and Stark broadening of the lines of radiation are taken into account as a function of vapor temperature and density for each vapor zone. Multigroup approximations (1000-4000 photon groups) were used for the continuum solution of the above equations. Plank averaging was usually used for the optically thin regions, whereas Rosseland averaging was more preferred for the optically thick regions [17]. Opacity and emissivity data are provided in the form of tables for a wide range of expected vapor densities and temperatures. The rate of energy loss due to radiation $Q_{r}$ in each zone can then be given by

$$
\mathrm{Q}_{\mathrm{r}}=\frac{1}{\rho} \sum_{v} \nabla \cdot\left(\mathrm{I}_{v}^{+}+\mathrm{I}_{v}^{-}\right)
$$

\section{Results}

The comprehensive self-consistent model described above is implemented in a new optimized version of the computer code $A^{*}$ THERMAL-S. In this analysis the thermal quench time during a disruption is assumed to be $100 \mu \mathrm{s}$. Other disruption times can easily be used. The calculations for the radiation transport and vapor hydrodynamics are extended up to $10 \mu$ s longer than the disruption time to realistically simulate a real situation, in which the vapor and radiation flux 
cannot suddenly disappear immediately after the disruption. Plasma energy densities of $10-100 \mathrm{MJ} / \mathrm{m}^{2}$ are used in this analysis. The effect of both plasma electrons and ions, with different kinetic energies, on the net erosion rate was investigated.

Figure 2 shows the time evolution of beryllium surface temperature, melt layer thickness, and eroded thickness for a disruption with $20 \mathrm{keV}$ plasma electrons and $10 \mathrm{MJ} / \mathrm{m}^{2}$ energy density. The sudden early rise in surface temperature is due to direct electron energy deposition in beryllium. The following decrease in the beryllium surface temperature was caused by the shielding effect of the eroded material above the beryllium surface. The flat temperature behavior during the rest of the disruption was mainly due to the low generated radiation heat flux in this case. The net ablation erosion rate for the given disruption conditions is about $4 \mu \mathrm{m}$, which is substantially lower than that without the shielding effect [13].

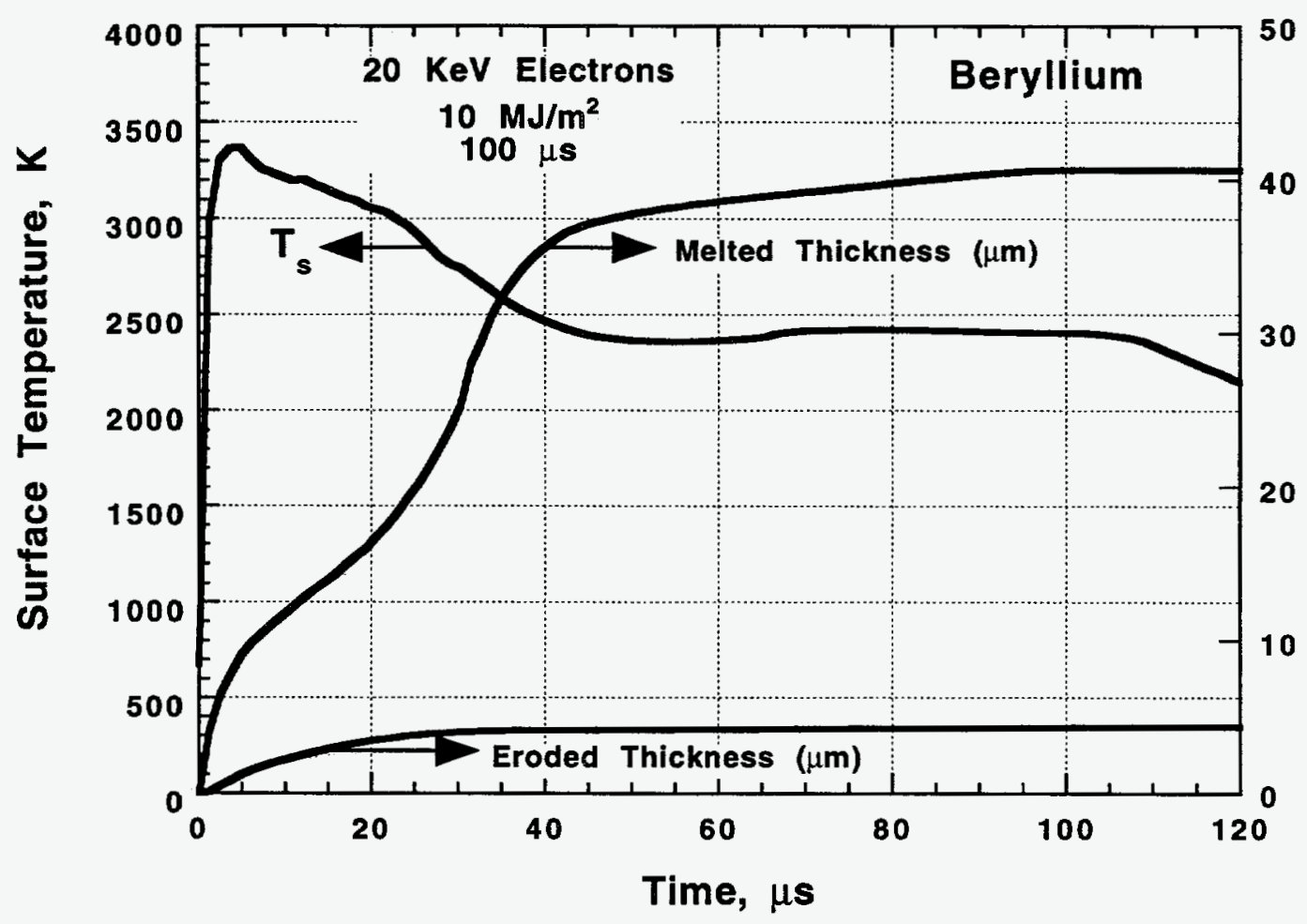

Fig. 2 Change in beryllium surface temperature, melt layer, and eroded thickness with time, following exposure to plasma electrons. 
Figure 3 shows the relationship of beryllium solid-liquid-vapor temperatures to distance at two times, i.e., $10 \mu \mathrm{s}$ and $100 \mu \mathrm{s}$, during the disruption. At an early time $(10 \mu \mathrm{s})$ the solid temperature was higher than at later times because of the direct deposition of plasma electrons. The temperature of the vapor layer at that time was relatively low $(\approx 1 \mathrm{eV})$ and the vapor layer only expanded to about $3-4 \mathrm{~cm}$ above the surface. At longer times, towards the end of the disruption, the solid-liquid temperature was lower because only a small fraction of the radiation energy was deposited on the surface. The vapor had then expanded to distances up to $70 \mathrm{~cm}$ above the surface and reached a temperature of $\approx 4 \mathrm{eV}$.

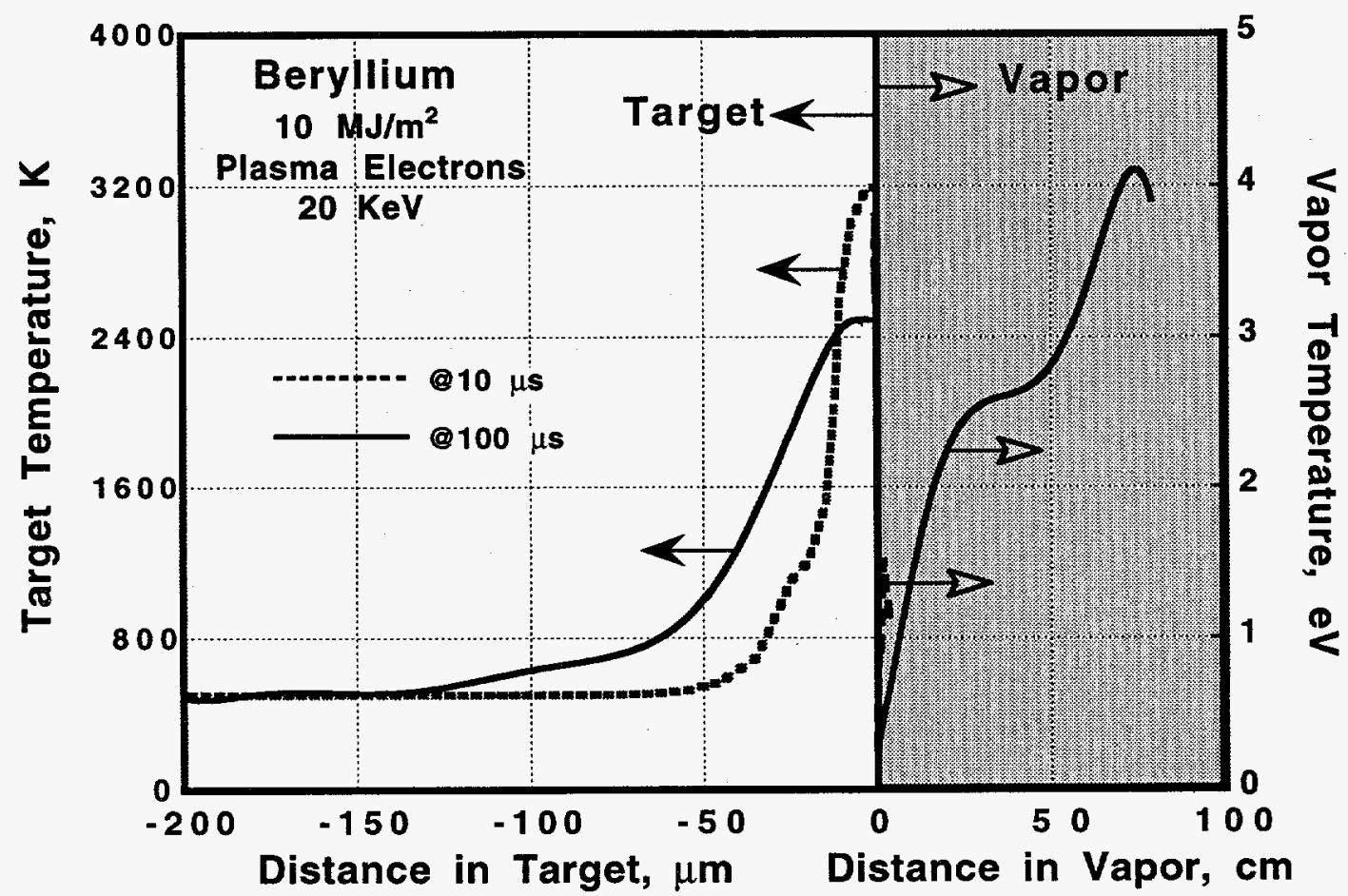

Fig. 3 Relationship of target and vapor temperature to distance at different times during disruption. 
It is still uncertain, however, what the plasma-particle kinetic energy would be at the divertor plate during a disruption. Estimates range from several keV down to few hundredths of an $\mathrm{eV}$. Figure 4 shows the effect of plasma-electron kinetic energy on the erosion rate. Particles with higher kinetic energy usually penetrate the target to a greater depth and result in more erosion rate. However, because more material is eroded by high-energy particles, the vapor, being optically thick in this case, will have a lower temperature and, consequently, emit less radiation flux toward the PFM. This is evident from the slope of ablated thickness in Fig. 4. Particles with lower kinetic energy erode less material and, for the same energy density, result in higher vapor temperatures and thus higher radiation fluxes.

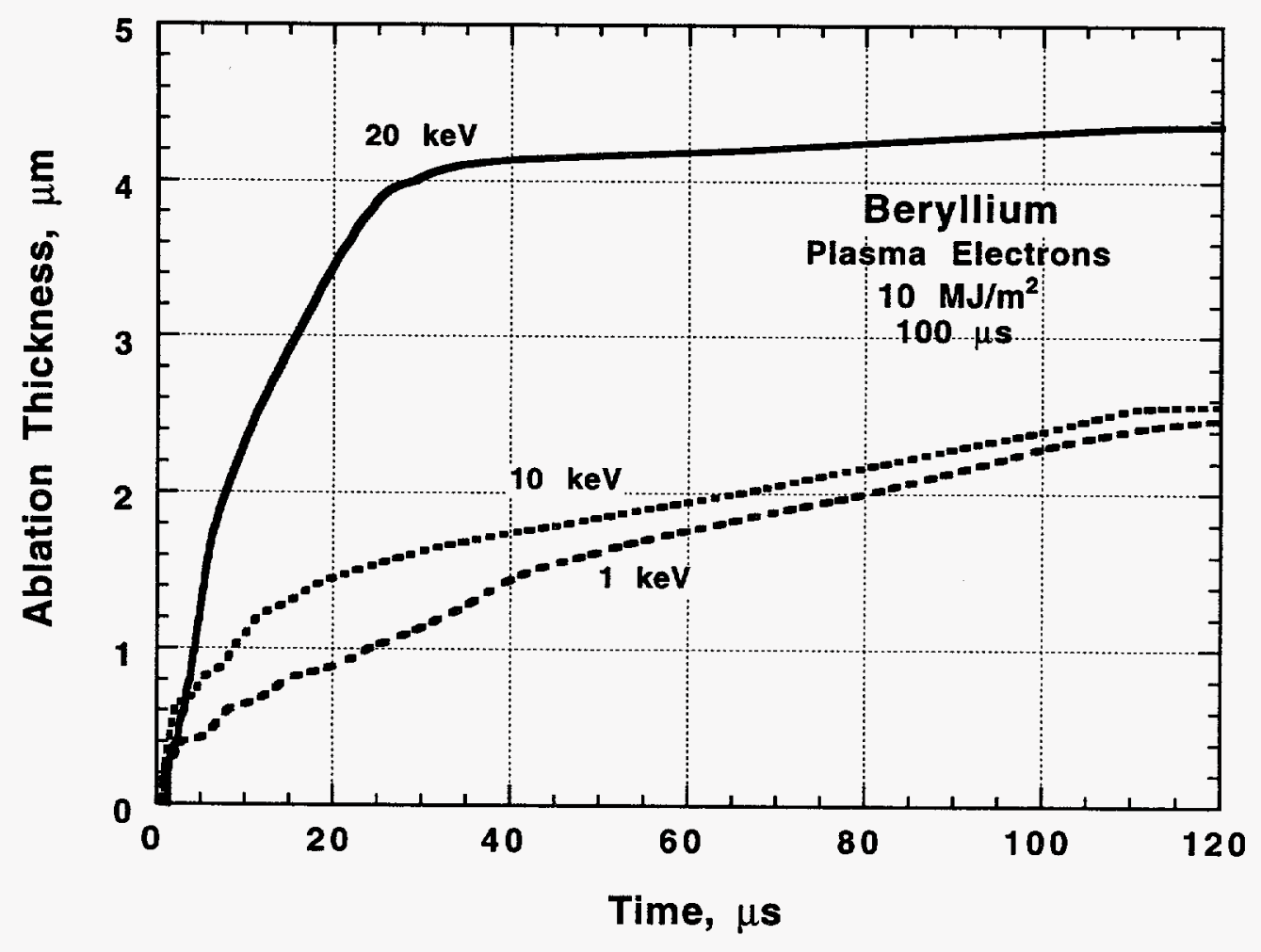

Fig. 4 Effect of plasma electron kinetic energy on beryllium erosion rate. 
Figure 5 shows the change in temperature of the beryllium vapor front. It can be seen that, in the case of $20 \mathrm{keV}$ plasma electrons, because of both the long range and the large mass of the eroded material, the temperature of the front vapor is only $\approx 3 \mathrm{eV}$. Particles with lower kinetic energy deposit their energy near the vapor front which results in higher front vapor temperature. This means that the vapor ionization rate is higher. Higher ionization rate means more free vapor electrons, which, in turn, result in a much shorter range of plasma electrons in the vapor because of their additional stopping power, etc. This is the main reason why the 1 and $10 \mathrm{keV}$ incident plasma electrons exhibit the same front vapor temperature, of $\approx 15 \mathrm{eV}$, halfway through the disruption.

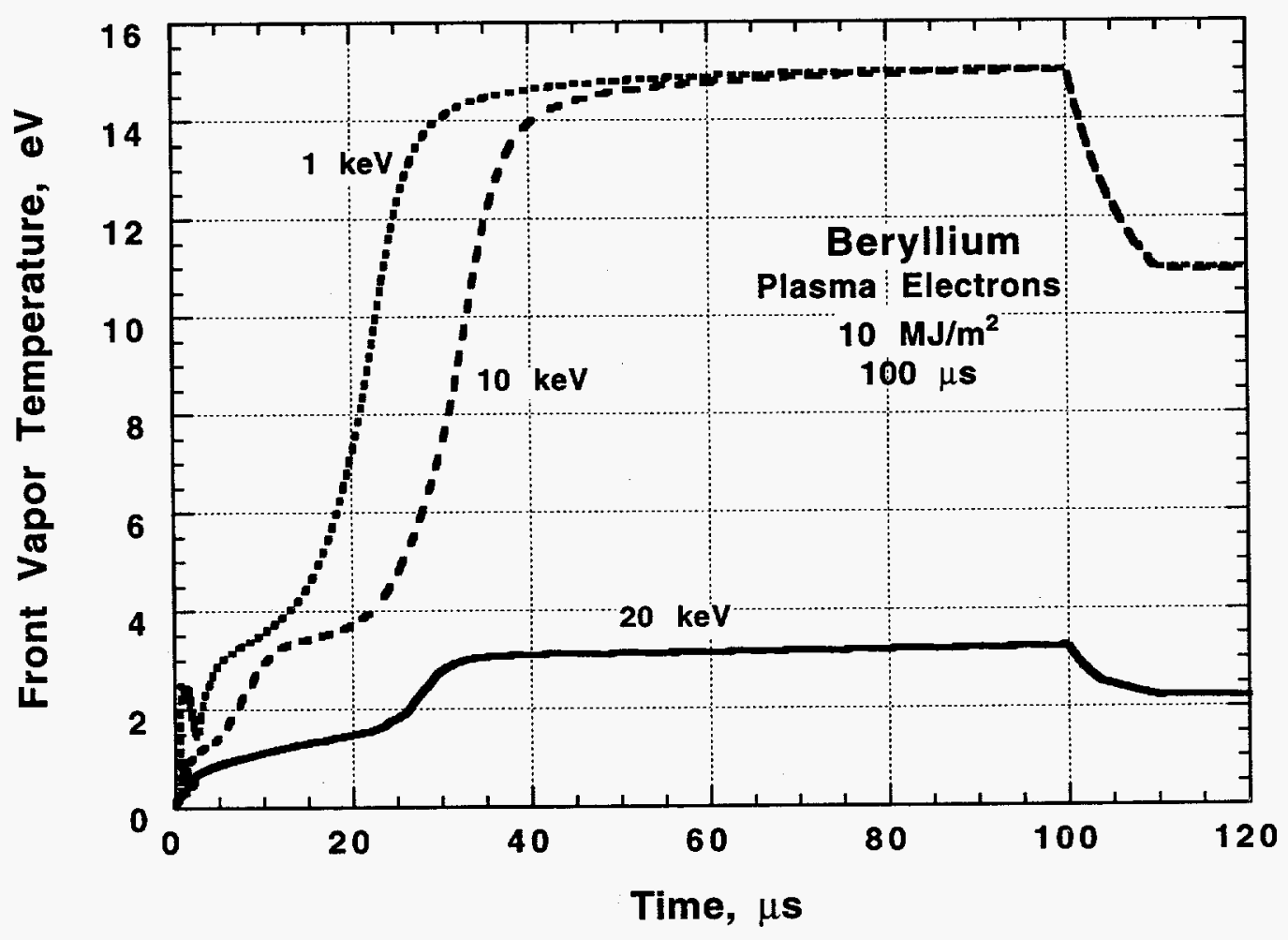

Fig. 5 Change in beryllium vapor front temperature with time. 
The difference between the effect of plasma electrons and plasma ions, with similar kinetic energy on the beryllium erosion rate is also studied. Basically, ions with higher kinetic energy $(20 \mathrm{keV})$ behave in a manner similar to that of electrons with lower kinetic energy $(1 \mathrm{keV})$, where the front vapor zone is heated to higher vapor temperatures and results in some radiation flux reaching the PFM surface. This is due to the fact that ions have much shorter range than electrons with the same kinetic energy. Ions then produce less erosion than electrons with similar kinetic energy. However, plasma ions exhibit a much larger momentum than electrons, a circumstance that tends to confine the vapor plasma closer to the PFM [21].

The energy density reaching the divertor plate during a disruption can be much higher than $10 \mathrm{MJ} / \mathrm{m}^{2}$. This can also occur if the plasma deposits its energy only on parts of the toroidal divertor plate. Figures 6 and 7 show the effect of higher energy densities (up to $100 \mathrm{MJ} / \mathrm{m}^{2}$ ) on the erosion rate of carbon and beryllium respectively. An order of magnitude increase in energy density resulted in an increase of only $\approx 30-40 \%$ in erosion rate. This is mainly because most of the incident energy was used to heat the front regions of the vapor, as shown in Figs. 8 and 9 for carbon and beryllium respectively. This means that most of the radiation flux is emitted in a direction away from the PFM. It can also be seen that, for the same heat flux, carbon erodes more than beryllium; this is because carbon radiates energy more than beryllium which results in more net radiation flux to the PFM and higher free-streaming energy flux from the near-wall vapor zone. In addition, the lower thermal conductivity of carbon has the effect of keeping the surface temperature higher which tends to increase the erosion. However, beryllium melts, and erosion of the melt layer during the disruption can be of serious concern [19]. Figure 10 shows carbon and beryllium erosion rates at various disruption energy densities. If the melt layer is lost during the disruption, beryllium erosion is about five times higher than that of carbon for the disruption conditions of this study. If the melt layer is lost as soon as it develops, however, disruption lifetime can be significantly reduced. More frequent repair or plasma-spraying will thus be required to maintain a reasonable divertor plate lifetime. 


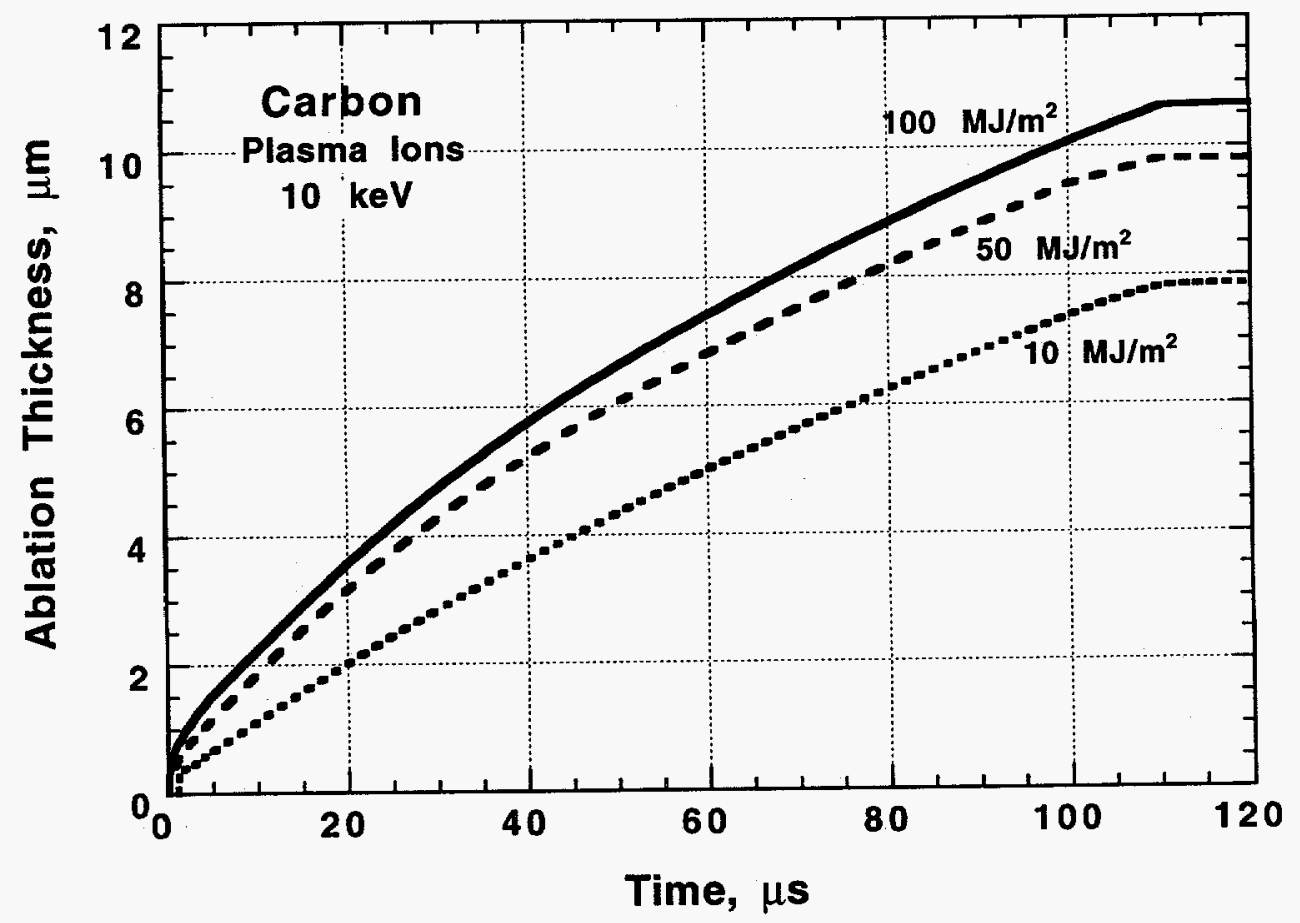

Fig. 6 Effect of higher disruption energy densities on carbon erosion rate.

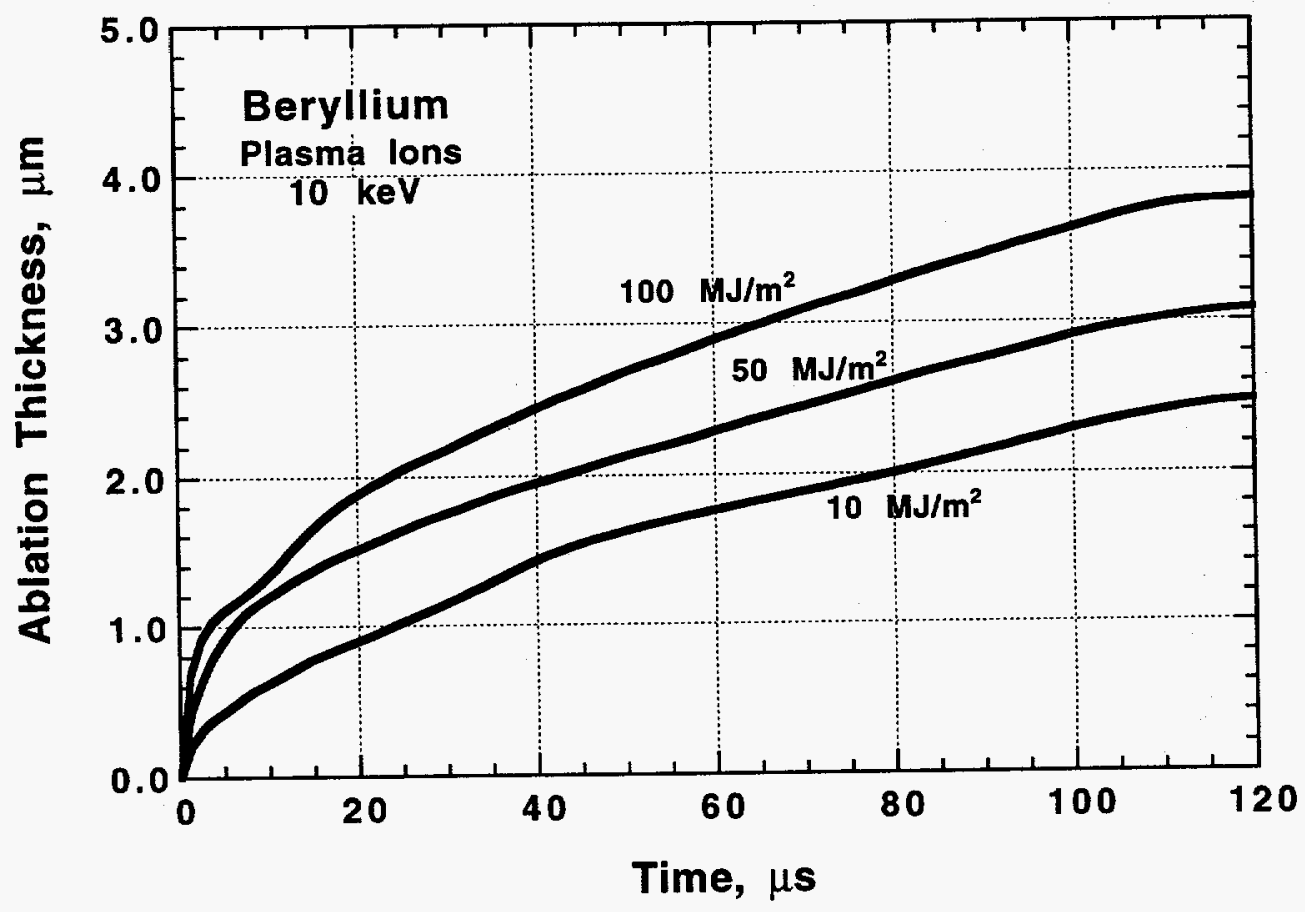

Fig. 7 Effect of higher disruption energy densities on beryllium erosion rate. 


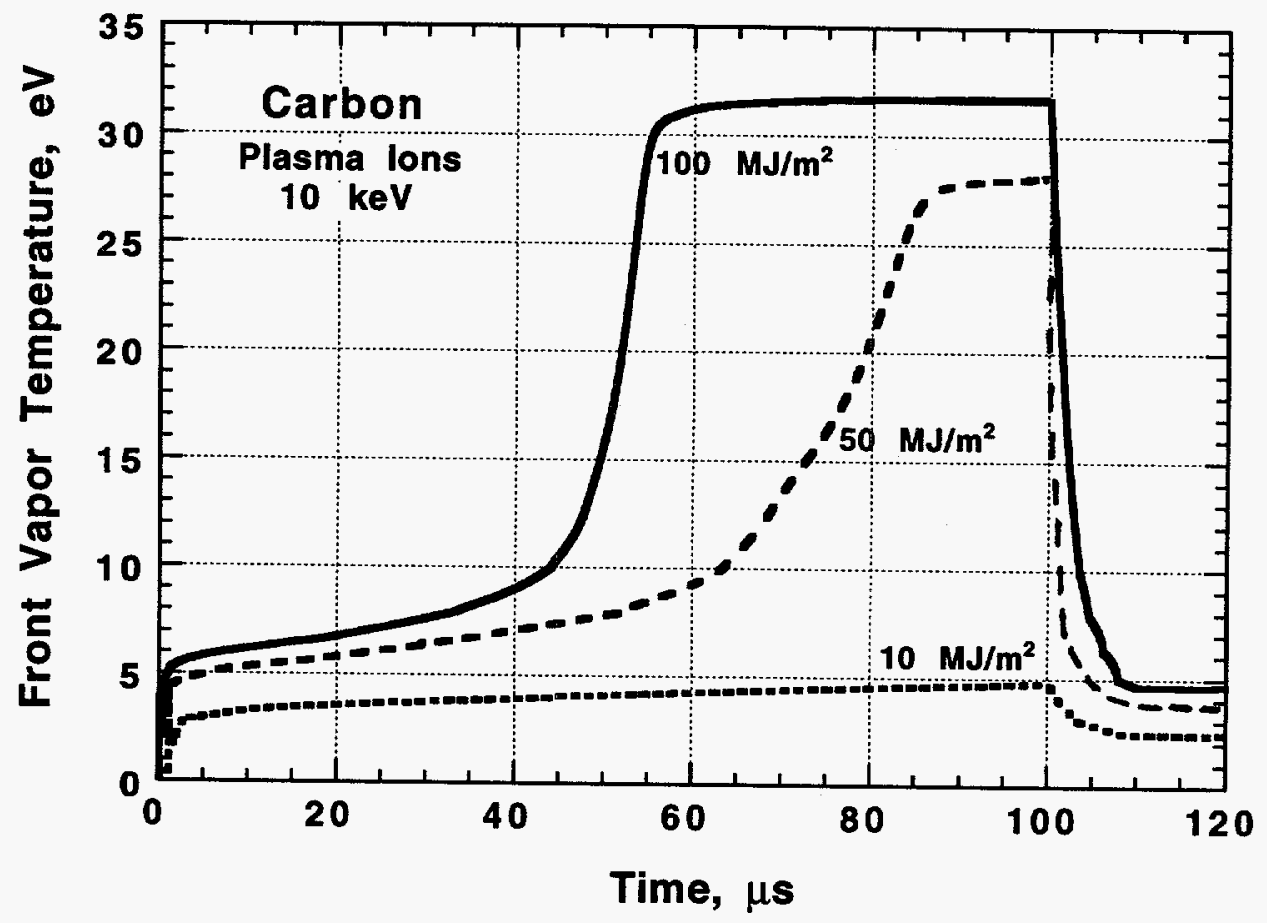

Fig. 8. Effect of higher disruption energy densities on carbon vapor-front temperature.

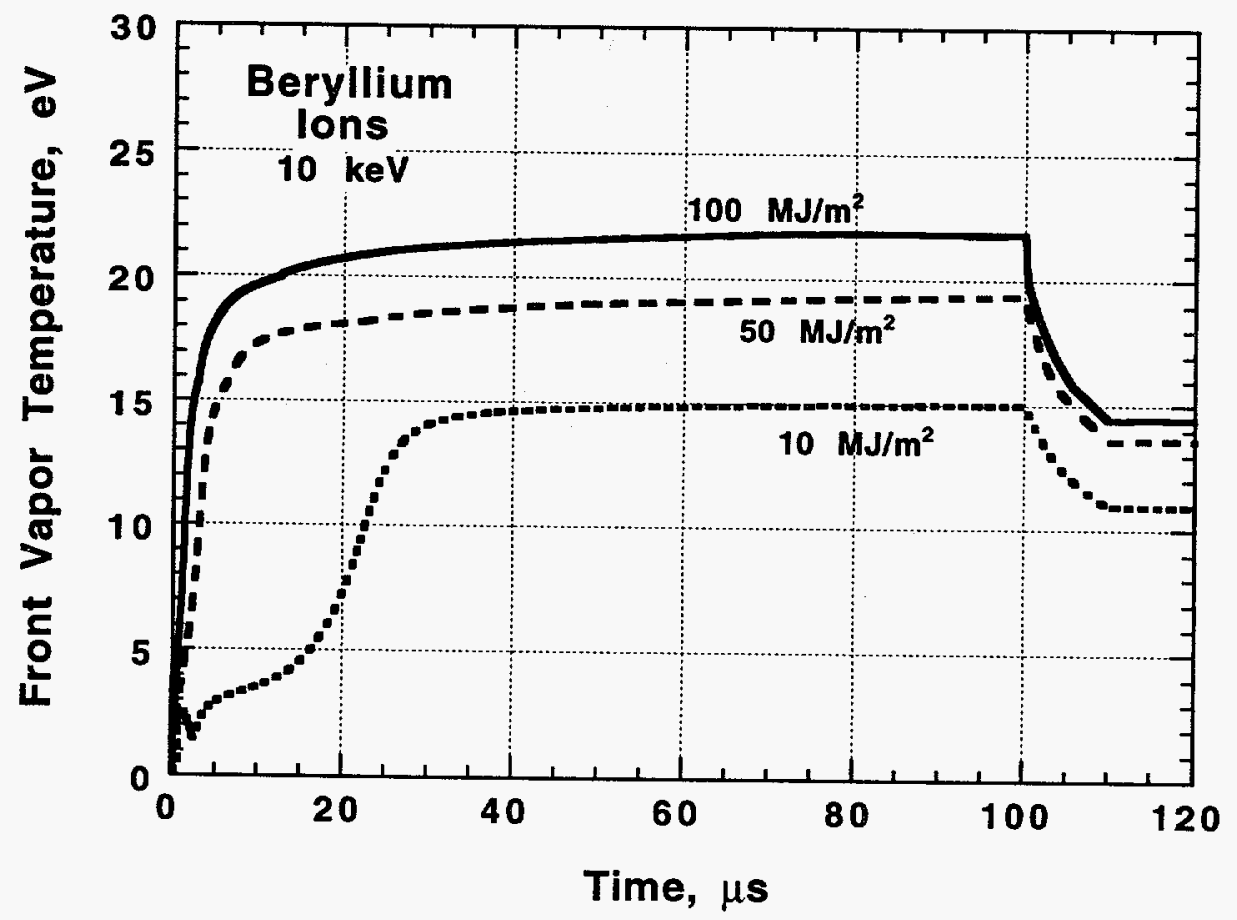

Fig. 9. Effect of higher disruption energy densities on beryllium vapor-front temperature. 


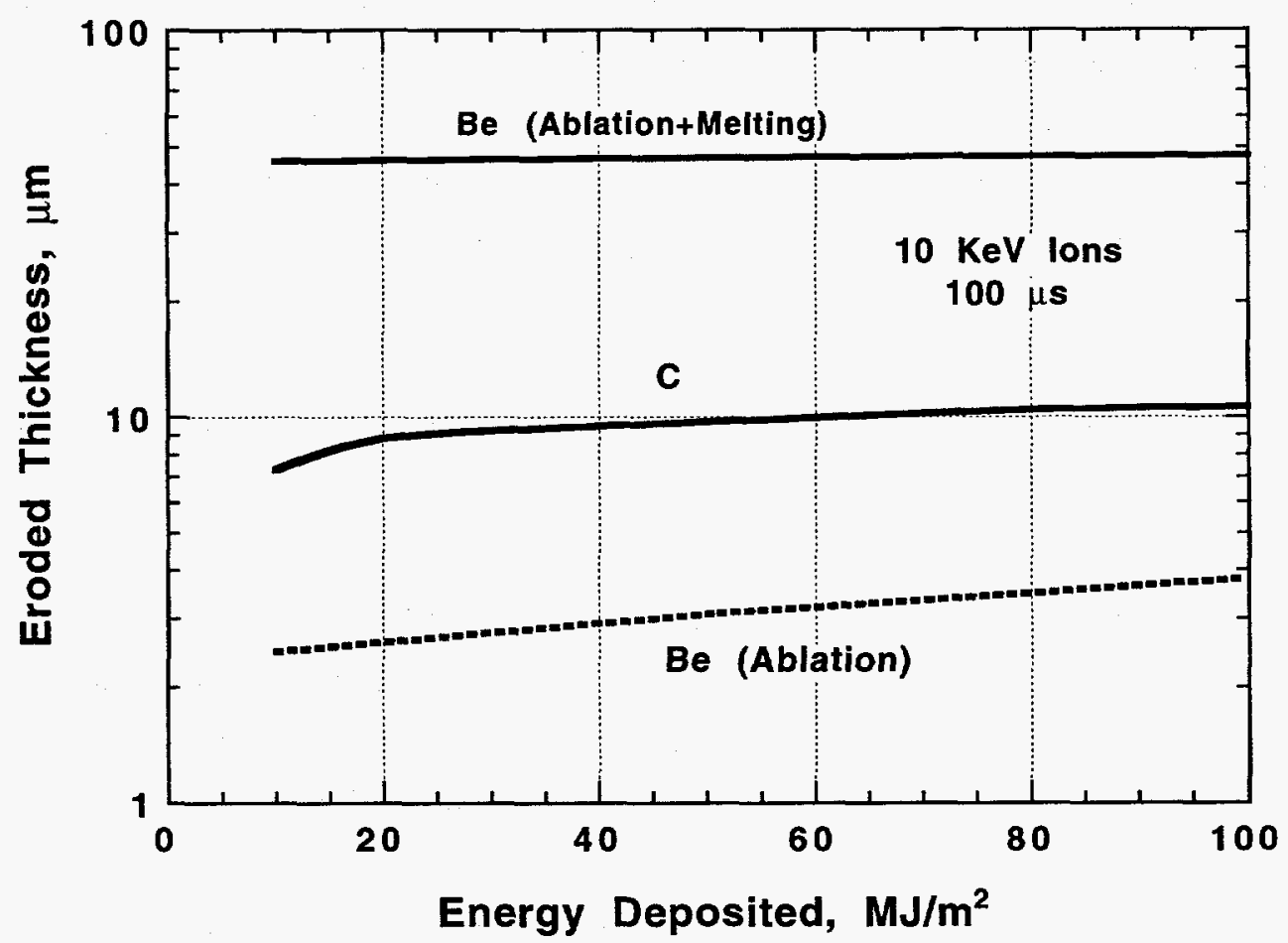

Fig. 10 Erosion rate of beryllium and carbon at various disruption energy densities.

Further analysis and investigation are needed for a number of important issues related to modeling erosion depth during a disruption. One important issue is the effect of an oblique magnetic field on the hydrodynamics of the vapor cloud that develops during a disruption. A 2-D magnetohydrodynamic model was recently developed and integrated with this model to study such an effect on the net erosion rate; preliminary analysis indicates that the magnetic field may reduce the overall erosion rate [20]. Another issue requiring investigation is the effect of a mixing zone between the incident plasma particles and the front vapor cloud. This zone can alter the radiative properties of the vapor zone, thus affecting the net radiative heat flux to the PFM. Another important factor that can affect erosion depth is the two-dimensional effects of radiation transport in the vapor cloud. A 2-D radiation transport model was recently developed and integrated with this model [21]. It is found that escaping lateral radiation from the vapor cloud can significantly reduce the net heat flux to the PFM, thus reducing the erosion rate. This is particularly important in disruption-simulation 
experiments where the exposed target area is very small compared to vapor expansion and the resulting erosion rates are low [21]. Vapor thermal conduction with turbulence and vapor instabilities may significantly increase heat flux to the PFM, resulting in a substantial increase in erosion rate. Erosion of melt layers that develop during a disruption also requires further analysis and study. Loss of the melt layer can severely shorten divertor plate lifetime.

\section{Conclusion}

A comprehensive self-consistent model was developed to take into account the various interaction zones/processes that occur during a disruption. The model realistically solves the problem of three moving boundaries, i.e., vapor expansion, surface recession, and liquid metal propagation front. Models for material thermal evolution with phase change, vapor hydrodynamics, and radiation transport, including line radiations, are integrated, perfected, and numerically optimized for realistic and efficient simulation of disruption effects.

Results of disruption simulation indicate that plasma electrons, will cause higher erosion rates than plasma ions with the same initial kinetic energy. Carbon ablation thickness is usually higher than that of beryllium under similar conditions. However, if the beryllium melt layer is lost during the disruption, beryllium erosion will be much higher than that of carbon-based materials. Higher disruption energy densities usually expend most of the energy in heating the front vapor, causing more forward radiation away from the PFM and less backward radiation towards the divertor. This scenario will generally result in a modest increase in erosion rate compared to an increase in energy density.

\section{Acknowledgment}

The authors sincerely thank Dr. G. Romanov, Dr. B. Bazilev, and Dr. V. Tolkach of the Institute of Heat and Mass Transfer at the Belorussia Academy of Science, Minsk, for their help in supplying the data on opacity and emissivity for this analysis.

\section{References}

[1] A. Sestero and A. Ventura, J. Nucl. Mater. $128 \& 129(1984) 828$.

[2] A. Hassanein et al., Nucl. Eng. Design/Fusion 1 (1984) 307. 
[3] B.J. Merrill and J.L. Jones, J. Nucl. Mater. 111 \& 112 (1982) 544.

[4] H. Bolt et al., J. Nucl. Mater. 196-198 (1992) 948.

[5] A. Hassanein and D. Ehst, J. Nucl. Mater. 196-198 (1992) 680.

[6] B. Goel et al., Fusion Technology (1992) 272.

[7] W. Höbel et al., J. Nucl. Mater. 196-198 (1992) 537.

[8] R.R. Peterson, Radiative heat transfer in self-shielding vapor layer during tokamak disruptions, UWFDM-357 (1983).

[9] A. Hassanein and D. Ehst, J. Nucl. Mater. (1994).

[10] J. Gilligan et al., J. Nucl. Mater. 162-164 (1989) 957.

[11] A. Hassanein, J. Nucl. Mater. 122 \& 123 (1984) 1453.

[12] A. Hassanein and D. Smith, J. Nucl. Mater. 191-194 (1992) 503.

[13] A. Hassanein, ASME, 88-WA/NE-2.

[14] R.J. Procassini and C.K. Birdsall, Phys. Fluids B3 (8) (1991) 1876.

[15] Y.L. Igitkhanov et al., Sov. I. Plasma Phys. 12 (1) 1986.

[16] B.N. Chetverushkin, Mathematical modelling of the radiative gas, Nauka, Moscow (1986).

[17] I.V. Nemchinov, "The Averaging of the Radiation Transport Equations for the Radiation Transport in Gas," VINITI, N1721-83, Moscow, 1983.

[18] S.I. Anisimov and A.Kh. Rakhmatulina, Sov. Phys. JETP, Vol. 37 (1973) 441. 
[19] A. Hassanein, Fusion Technology 15 (1989) 513.

[20] A. Hassanein and I. Konkashbaev, "An Assessment of Disruption Erosion in the ITER Environment," presented at the Third International Symposium on Fusion Nuclear Technology (ISFNT-3), Los Angeles, California, June 27 July 1, 1994. To be published in Fusion Engineering/Design.

[21] A. Hassanein, "Plasma disruption modeling and simulations," invited paper presented at the 11th Topical Meeting on the Technology of Fusion Energy, ANS, June 19-23, 1994. To be published in Fusion Technology. 


\section{DISTRIBUTION LIST FOR ANL/FPP/TM-271}

\section{Internal}

H. Attaya

A. Hull

S. Bhattacharyya

C. Johnson

W. Shack

M. Billone

T. Kassner

Dale L. Smith

J. Brooks

M. Lineberry

D.-K. Sze

D. Diercks

S. Majumdar

S.W. Tam

D. Ehst

R. Mattas

Y. Gohar

B. Micklich

L. Turner

D. Gruen

K. Natesan

A. Hassanein (10)

J.H. Park

H. Herman

B. Picologlou

T. Hua

R. Poeppel

T. Yule

FPP Files (10)

TIS Files (1)

\section{External}

DOE/OSTI for distribution per UC-423 (48)

Manager, Chicago Operations Office

ANL-E Libraries (2)

ANL-W Library

M. Abdou, University of California, Los Angeles

M. Akiba, Japan Atomic Energy Research Institute, Japan

$J$. Anderson, Los Alamos National Laboratory

C. Baker, University of California, San Diego

J. Bartlit, Los Alamos National Laboratory

S. Berk, U.S. Department of Energy

H. Bolt, Kernforschungszentrum Karlsruhe, Germany

L. Bühler, Kernforschungszentrum Karlsruhe, Germany

T. Burchell, Oak Ridge National Laboratory

R. Causey, Sandia National Laboratories, Livermore

M. Cohen, U.S. Department of Energy

S. Cohen, Princeton Plasma Physics Laboratory

C. Croessman, Sandia National Laboratories, Albuquerque

W. Daenner, ITER, Germany

J. Davis, McDonnell Douglas Astronautics Company

J. Doggett, Lawrence Livermore National Laboratory

W. Gauster, ITER, JCT, Garching, Germany

D. Gelles, Pacific Northwest Laboratories

N. Ghoneim, University of California, Los Angeles

L. Greenwood, Battelle Pacific Northwest Laboratory

T. James, U.S. Department of Energy

I. Konkashbaev, Troitsk Institute for Innovation, Russia

V. Kozhevin, Efremov Institute, St. Petersburg, Russia

D. Lousteau, Fusion Engineering Design Center

H. Maekawa, Japan Atomic Energy Research Institute, Japan

S. Malang, Kernforschungszentrum Karlsruhe, Germany

Y. Martynenko, Kurchatov Institute, Russia

R. McGrath, Sandia National Laboratories, Albuquerque 
D. Muntz, Kernforschungszentrum Karlsruhe, Germany

H. Nickel, Kernforschungszentrum Karlsruhe, Germany

R. Nygren, Sandia National Laboratories, Albuquerque

A. Opdenaker, U.S. Department of Energy

R. Parker, ITER JCT, Garching, Germany

S. Piet, ITER JCT, San Diego, California

D. Post, ITER JCT, San Diego, California

R. Price, U.S. Department of Energy

A. Raffray, ITER JCT, Garching, Germany

M. Sawan, University of Wisconsin

K. Schultz, General Atomic

M. Seki, Japan Atomic Energy Research Institute, Japan

D. Steiner, Rensselaer Polytechnic Institute

I. Sviatoslavsky, University of Wisconsin

M. Tillack, University of California, San Diego

M. Ulrickson, Sandia National Laboratory, Albuquerque

J. Van der Laan, ECN, Netherlands

J. Vetter, Kernforschungszentrum Karlsruhe, Germany

G. Vieider, ITER, Germany

R. Watson, Sandia National Laboratories, Albuquerque

F.W. Wiffen, U.S. Department of Energy

K. Wilson, Sandia National Laboratories, Livermore

$M$. Youssef, University of California, Los Angeles

Bibliothek, Max-Planck-Institute fur Plasmaphysik, Germany

C.E.A. Library, Fontenay-aux-Roses, France

Librarian, Culham Laboratory, England

Thermonuclear Library, Japan Atomic Energy Research Institute, Japan 